\title{
Myeloma-derived macrophage inhibitory factor regulates bone marrow stromal cell-derived IL-6 via c-MYC
}

\author{
Rachel E. Piddock', Christopher R. Marlein', Amina Abdul-Aziz', Manar S. Shafat ${ }^{1}$, Martin J. Auger², \\ Kristian M. Bowles ${ }^{1,2^{*+}}$ and Stuart A. Rushworth ${ }^{1 *+}$
}

\begin{abstract}
Multiple myeloma (MM) remains an incurable malignancy despite the recent advancements in its treatment. The protective effects of the niche in which it develops has been well documented; however, little has been done to investigate the MM cell's ability to 're-program' cells within its environment to benefit disease progression. Here, we show that MM-derived macrophage migratory inhibitory factor (MIF) stimulates bone marrow stromal cells to produce the disease critical cytokines IL-6 and IL-8, prior to any cell-cell contact. Furthermore, we provide evidence that this IL-6/8 production is mediated by the transcription factor CMYC. Pharmacological inhibition of CMYC in vivo using JQ1 led to significantly decreased levels of serum IL-6-a highly positive prognostic marker in MM patients.

Conclusions: Our presented findings show that MM-derived MIF causes BMSC secretion of IL-6 and IL-8 via BMSC CMYC. Furthermore, we show that the CMYC inhibitor JQ1 can reduce BMSC secreted IL-6 in vivo, irrespective of tumor burden. These data provide evidence for the clinical evaluation of both MIF and CMYC inhibitors in the treatment of MM.
\end{abstract}

Keywords: Myeloma, MIF, CMYC, BMSC, Stromal, IL-6, IL-8, Bone marrow

Despite significant recent advancements made in the treatment of multiple myeloma (MM), relapse remains inevitable and the disease presently remains incurable. This is attributable, in part, to the highly protective nature of the BM micro-environment niche in which the malignant plasma cells proliferate. Macrophage migratory inhibitory factor (MIF) is a cytokine associated with various roles [1] and is rapidly developing a pro-tumoral identity [2]. Elevated MIF levels are described in MM and have been implicated in MM bone marrow homing and chemotherapy resistance [3]; however, the adaptive effect that MM-derived MIF has on the tumor microenvironment is not yet defined. Here, we investigate the function of MM-derived MIF in the MM microenvironment by examining its effects on bone marrow stromal cells (BMSC).

\footnotetext{
* Correspondence: kristian.bowles@nnuh.nhs.uk; s.rushworth@uea.ac.uk ${ }^{\dagger}$ Kristian M. Bowles and Stuart A. Rushworth contributed equally to this work. ${ }^{1}$ Department of Molecular Haematology, Norwich Medical School, The University of East Anglia, Norwich Research Park, Norwich NR4 7TJ, UK Full list of author information is available at the end of the article
}

We and others have found that MM cells have significantly elevated MIF gene expression and secreted protein levels [3] (Additional file 1: Figure S1). MIF KD resulted in lower MM proliferation in BMSC co-culture (Additional file 1: Figure S2C) alongside reduced tumor burden and improved overall survival in vivo (Fig. 1a, b). To investigate the effects of MIF secretion by MM on its microenvironment, we used cytokine arrays to establish if cytokine changes occur when MM cells are cultured with primary BMSC. Elevated levels of IL-6/8 were detected in co-culture experiments when compared with either BMSC or MM monoculture arrays (Fig. 1c, d); no MIF was detected in BMSC cultured alone. Cytokine array analysis of the supernatant from MIF-stimulated BMSCs confirmed this IL-6/IL-8 secretion (Fig. 1e, f) and was quantified via ELISA (Additional file 2) in Fig. 1g. Pretreatment of BMSC with the MIF inhibitor ISO-1 significantly reduced the MIF induction of IL-6/IL- 8 by BMSC (Fig. 1h). BMSC expressed all three known receptors for MIF (CXCR4, CXCR2 and CD74); however, only blocking

(c) The Author(s). 2018 Open Access This article is distributed under the terms of the Creative Commons Attribution 4.0 International License (http://creativecommons.org/licenses/by/4.0/), which permits unrestricted use, distribution, and reproduction in any medium, provided you give appropriate credit to the original author(s) and the source, provide a link to the Creative Commons license, and indicate if changes were made. The Creative Commons Public Domain Dedication waiver (http://creativecommons.org/publicdomain/zero/1.0/) applies to the data made available in this article, unless otherwise stated. 
a

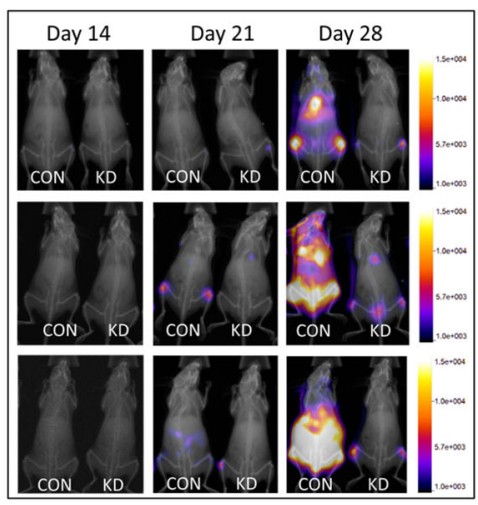

C

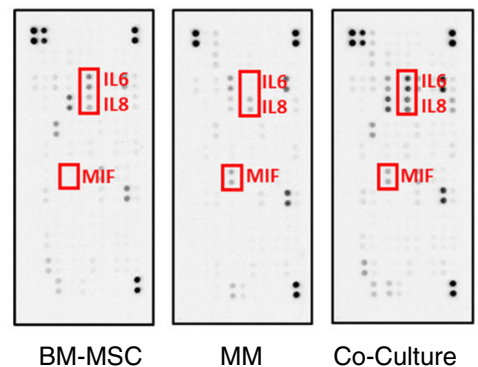

e
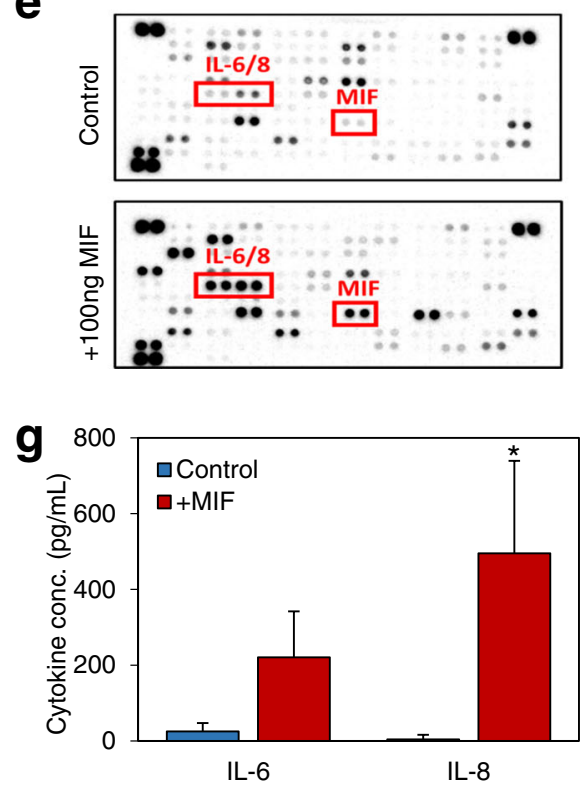

b

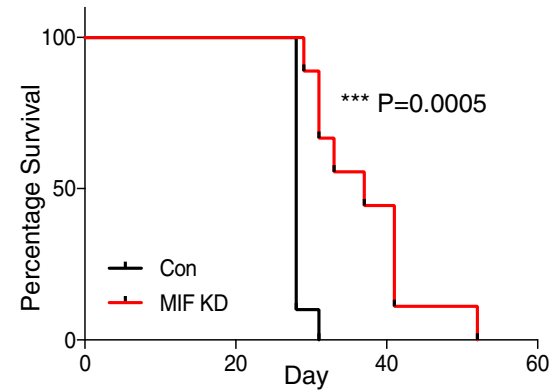

d

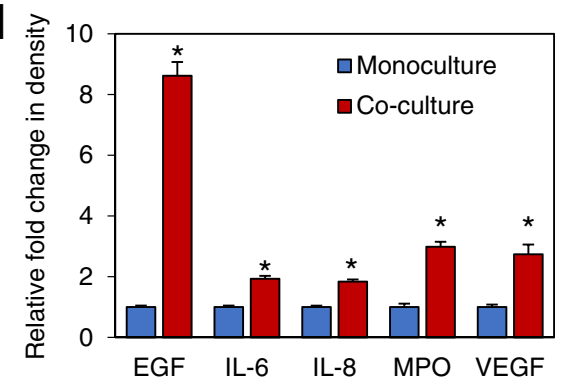

f

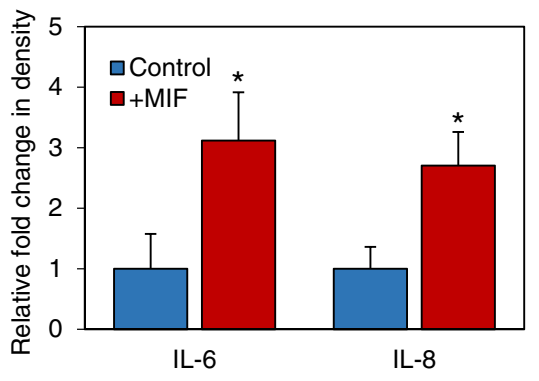

h

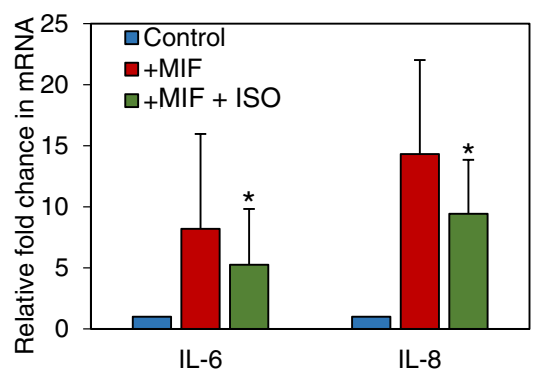

Fig. 1 MM derived MIF is pro-tumoral and drives BMSC IL-6 and IL-8. $1 \times 10^{6}$ MM.1S-luc cells (ShE control $n=10$, and ShMIF $n=7$ ) were injected via the tail vein of 6-8-week-old NSG mice. a Mice were monitored weekly by bioluminescent imaging. b Kaplan-Meier curve showing survival, analyzed using Mantel Cox regression. c Representative $(n=3)$ Human XL cytokine array output after a 24-h incubation in either mono or co-culture, cell supernatant was used for analysis. $\mathbf{d}$ Graphical representation of $\mathbf{c}$ - values for BMSC and MM monoculture intensities were added together and were analyzed against co-culture experiment signal intensity using $\mathrm{HL}++$ image software which show differences in several key cytokines. e, $\mathbf{f}$ BMSC were stimulated with $100 \mathrm{ng} / \mathrm{mL}$ of human recombinant MIF and incubated for $24 \mathrm{~h}$; supernatant was used for assay. Representative $(n=3)$ image of cytokine array (e) and subsequent graphical representation ( $\mathbf{f})$ of analysis using $\mathrm{HL}++$ software. $\mathbf{g}$ Primary BMSC $(n=4)$ were stimulated with $100 \mathrm{ng} / \mathrm{mL}$ recombinant human MIF for $6 \mathrm{~h}$ after which IL-6 and IL-8 protein excretion was analyzed by ELISA. $\mathbf{h}$ Primary BMSC $(n=4)$ were incubated with/ without $10 \mu \mathrm{g}$ ISO-1 and then stimulated with $100 \mathrm{ng} / \mathrm{mL}$ recombinant human MIF for $6 \mathrm{~h}$. IL-6 and IL-8 transcriptional levels were then analyzed by RT-PCR 
CD74 inhibited MIF-Induced IL6/8 (Additional file 1: Figure S3A\&B).

An inhibitor panel was used to screen for potential pathways responsible for MIF-induced BMSC-derived IL-6/IL-8 expression, and we found that JQ1 inhibited MIF induced IL-6 and IL-8 (Fig. 2a, b) mRNA in BMSC. We then determined if JQ1 could regulate BMSC protumoral interleukin production in-vivo. Although BMSC are often cyto-protective in the context of anti-MM therapy, others have observed that the sensitivity of MM cell lines to JQ1 was unchanged by the presence of HS-5 [4]. Following MM engraftment (Fig. 2d), mice were randomized and treated for 5 days with I.P injections of $50 \mathrm{mg} / \mathrm{kg}$ JQ1 or alternatively vehicle control. Under
7 days of treatment with JQ1 was predicted to have no measurable effect on tumor burden [4] and was selected to control for the effects of tumor burden on MM-MIF secretion and subsequent BMSC IL-6 expression. We found no difference in MM burden between groups (Fig. 2d, f). Nevertheless, murine IL-6 was significantly reduced in the JQ1-treated animals (carrying human MM) compared to control animals (Fig. 2e).

Despite MIF's association with hematological malignancies, previous work has focused on the effects of MIF on the malignant cells rather than the supportive cells of the microenvironment [3]. Here, we show that MM-derived MIF is pro-tumoral through induction of BMSC-derived IL-6/8. IL-6 is central to MM a

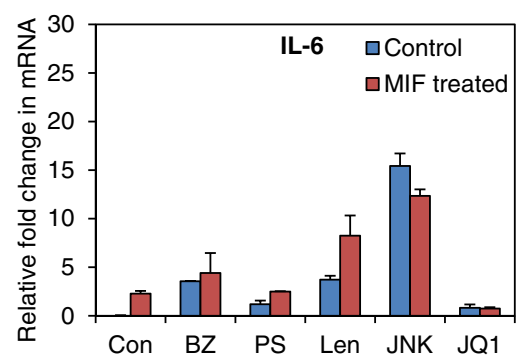

C

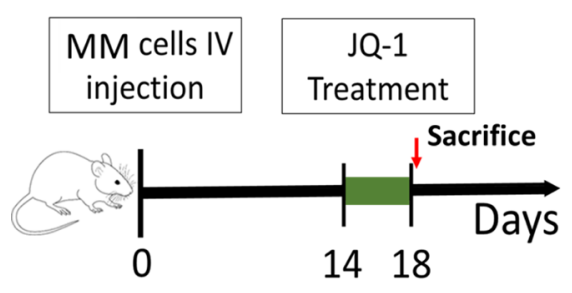

e

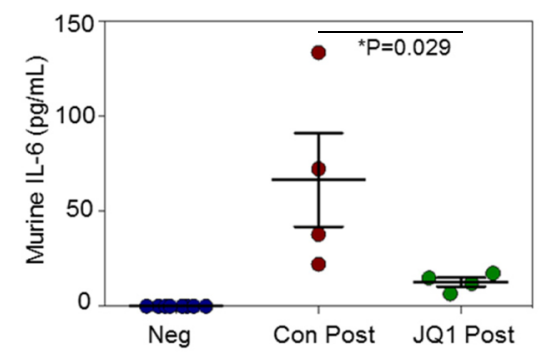

b

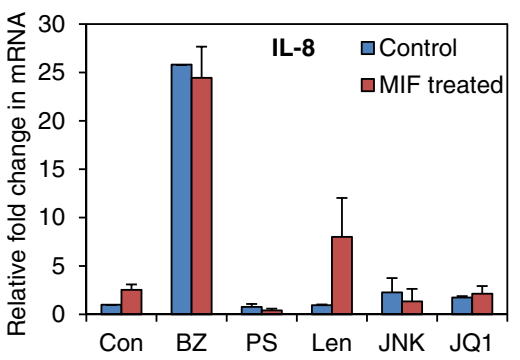

d

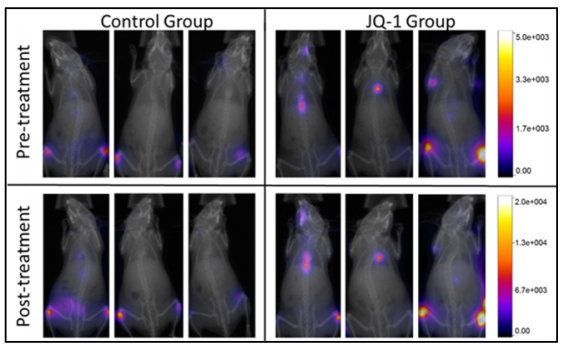

f

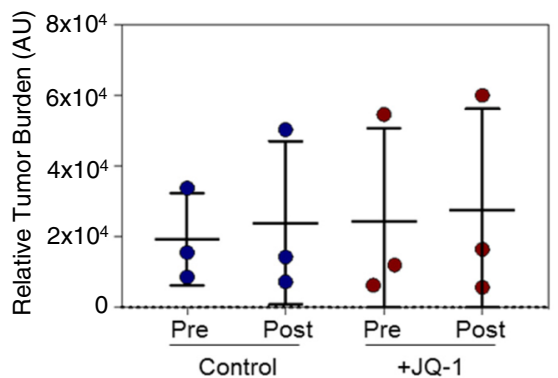

Fig. 2 MM-derived MIF regulates bone marrow stromal cell-derived IL-6 and IL-8 via CMYC. a, b BMSC cells were pretreated with various drugs (bortezomib 10 nM, PS341 100 nM, Lenolidiomide 500 nM, JNKV 10uM, and JQ1 500 nM) for 30 mins and then activated with MIF for 2 h. RNA was extracted, and transcriptional levels of IL-6 (a) or IL-8 (b) was analyzed. c Schematic showing JQ1 in vivo experiment. $1 \times 10^{6}$ U266 cells were injected via the tail vein of NGS mice $(n=8)$. Following a 2-week engraftment period, mice were treated with 50 mg/kg JQ1 or vehicle control daily for 5 days, after which all mice were sacrificed. $\mathbf{d}$ Mice were monitored pre and post treatment with JQ1 by bioluminescent imaging which was quantified (f) by ImageJ densitometry. e ELISA data showing murine IL-6 serum concentration following JQ1 treatment. Baseline levels of murine IL-6 were non-detectable in mice without MM 
pathogenesis and primarily comes from the BMSC in the tumor micro-environment [5]. Here, we place IL-6 downstream of MIF-induced BMSC activation [6, 7]. IL8 expression in BMSC, which has been shown to parallel MM disease progression [8] and positively influence osteoclastogenesis in MM [9], was increased in BMSC in response to MIF. Furthermore, the BET-bromodomain inhibitor JQ1 significantly decreased IL-6/8 secretion in MIF-stimulated BMSC. In vivo use of JQ1 significantly reduced levels of murine IL- 6 in the serum $[5,10,11]$. Taken together, this suggests that JQ1 is exerting antiMM activity, in part, through a direct effect on BMSC via the inhibition of BMSC IL-6 (and IL-8) synthesis. This in turn could explain why BMSC do not appear to offer MM protection from JQ1 therapy.

\section{Additional files}

Additional file 1: Figure S1. (A) Relative transcriptional levels of MIF expression in B cells, T cells, non-malignant plasma cells and CD138+ purified primary MM cells were normalized to Beta-Actin $(n=5)$. (B) MIF ELISA data showing the increase in MIF extracellular protein levels in CD138+ purified primary MM cells and MM cell lines in comparison to other cell types $(n=5)$. Figure S2. (A) MM.1 s cells were transduced with lentivirus targeted to MIF or control shRNA for $96 \mathrm{~h}$. RNA was extracted and MIF mRNA expression was analyzed via RT-PCR to confirm KD. (B) Cells described in (A) were cultured for $24 \mathrm{~h}$ in fresh media after which the supernatant was analyzed for MIF via ELISA. (C) MM.1S MIF KD cells were co-cultured with primary BMSC for $96 \mathrm{~h}$, after which MM cells were analyzed using Cell Titre Glo (CTG), normalized to a ShE control. Figure S3. (A) BMSC from MM patients was analyzed for CXCR4, CXCR2 and CD74 using flow cytometry and compared to isotype control. (B) BMSC were preincubated with either AMD3100 (10 $\mu \mathrm{M})$, SB225002 (100 nM) and anti-CD74 $(10 \mu \mathrm{g} / \mathrm{ml})$. BMSC were then stimulated with recombinant MIF (100 ng/ml) for $2 \mathrm{~h}$. RNA was extracted and analyzed for IL-6 and IL-8 expression using Real-time PCR. (PPTX $178 \mathrm{~kb}$ )

Additional file 2: Supplementary methods. Methods section. (DOCX 19 kb)

\section{Abbreviations}

BMSC: Bone marrow stromal cell; IL: Interleukin; MIF: Macrophage migratory inhibitory factor; MM: Multiple myeloma; NSG: NOD.Cg-Prkdcscid IL2rgtm1Wj//Sz

\section{Acknowledgements}

The authors wish to thank The Norwich Research Park (NRP), BBSRC, The National Institutes for Health Research (UK), The Big C, and The Rosetrees Trust for funding. Additionally, we are grateful to Professor Richard Ball and lain Sheriffs, Norwich tissue bank (UK) for help with sample collection. pCDHluciferase-T2A-mCherry was kindly gifted from Prof. Dr. med. Irmela Jeremias, Helmholtz Zentrum München, Munchen, Germany.

\section{Funding}

REP receives funding from the Norwich Research Park doctoral training program partnership, which is supported by the BBSRC. CRM is funded by the Rosetrees Trust, MSS by The Big C, and MJA and KMB are supported by the Norwich and Norfolk University Hospital. SAR receives funding from the University of East Anglia.

\section{Availability of data and materials}

The datasets supporting the conclusions of this article are included within this article and additional files.

\section{Authors' contributions}

REP, AA, KMB and SAR designed the research; REP, MSS and SAR performed the research; REP and CRM carried out in vivo work; MJA and KMB provided essential knowledge and reagents; REP, KMB and SAR wrote the paper. All authors read and approved the final manuscript.

\section{Ethics approval and consent to participate}

Informed consent was given in accordance with the Declaration of Helsinki and under approval from the Health Research Authority of the National Health Service, United Kingdom (07/H0310/146).

All animal experiments were performed in accordance with UK Home Office and University of East Anglia Animal Welfare Ethics Review Board regulations.

\section{Consent for publication}

Informed consent has been obtained from all patients (see above)

\section{Competing interests}

The authors declare that they have no competing interests.

\section{Publisher's Note}

Springer Nature remains neutral with regard to jurisdictional claims in published maps and institutional affiliations.

\section{Author details}

${ }^{1}$ Department of Molecular Haematology, Norwich Medical School, The University of East Anglia, Norwich Research Park, Norwich NR4 7TJ, UK. ${ }^{2}$ Department of Haematology, Norfolk and Norwich University Hospitals NHS Trust, Colney Lane, Norwich NR4 7UY, UK.

Received: 21 February 2018 Accepted: 6 May 2018

Published online: 16 May 2018

\section{References}

1. Lue H, Kleemann R, Calandra T, Roger T, Bernhagen J. Macrophage migration inhibitory factor (MIF): mechanisms of action and role in disease. Microbes Infect. 2002:4(4):449-60.

2. O'Reilly C, Doroudian M, Mawhinney L, Donnelly SC. Targeting MIF in cancer: therapeutic strategies, current developments, and future opportunities. Med Res Rev. 2016;36(3):440-60. PubMed PMID: 26777977. Epub 2016/01/19. eng

3. Zheng Y, Wang Q, Li T, Qian J, Lu Y, Li Y, et al. Role of myeloma-derived MIF in myeloma cell adhesion to bone marrow and chemotherapy response. J Natl Cancer Inst. 2016;108(11):djw131.

4. Delmore JE, Issa GC, Lemieux ME, Rahl PB, Shi J, Jacobs HM, et al. BET bromodomain inhibition as a therapeutic strategy to target c-Myc. Cell. 2011;146(6):904-17. PubMed PMID: PMC3187920

5. Mahtouk K, Moreaux J, Hose D, Reme T, Meissner T, Jourdan M, et al. Growth factors in multiple myeloma: a comprehensive analysis of their expression in tumor cells and bone marrow environment using Affymetrix microarrays. BMC Cancer. 2010;10:198. PubMed PMID: 20465808. Pubmed Central PMCID: PMC2882921. Epub 2010/05/15. eng

6. Klein B, Zhang X, Lu Z, Bataille R. Interleukin-6 in human multiple myeloma. Blood. 1995:85(4):863-72.

7. Hilbert DM, Kopf M, Mock BA, Kohler G, Rudikoff S. Interleukin 6 is essential for in vivo development of B lineage neoplasms. J Exp Med. 1995;182(1): 243-8. PubMed PMID: 7790819. Pubmed Central PMCID: PMC2192088. Epub 1995/07/01. eng

8. Kline M, Donovan K, Wellik L, Lust C, Jin W, Moon-Tasson L, et al. Cytokine and chemokine profiles in multiple myeloma; significance of stromal interaction and correlation of $\mathrm{IL}-8$ production with disease progression. Leuk Res. 2007;31(5):591-8.

9. Bendre MS, Montague DC, Peery T, Akel NS, Gaddy D, Suva LJ. Interleukin-8 stimulation of osteoclastogenesis and bone resorption is a mechanism for the increased osteolysis of metastatic bone disease. Bone. 2003;33(1):28-37.

10. Jourdan M, Mahtouk K, Veyrune J-I, Couderc G, Fiol G, Redal N, et al. Delineation of the roles of paracrine and autocrine interleukin-6 (IL-6) in myeloma cell lines in survival versus cell cycle. A possible model for the cooperation of myeloma cell growth factors. Eur Cytokine Netw. 2005;16(1): 57-64.

11. Dankbar B, Padró T, Leo R, Feldmann B, Kropff M, Mesters RM, et al. Vascular endothelial growth factor and interleukin-6 in paracrine tumor-stromal cell interactions in multiple myeloma. Blood. 2000;95(8):2630-6. 